\title{
Modes of comparison and Question under Discussion: Evidence from 'contrastive comparison' in Japanese*
}

\author{
Yusuke Kubota \\ University of Tokyo, JSPS
}

\author{
Ai Matsui \\ Michigan State University
}

\begin{abstract}
Based on new empirical evidence from Japanese, we first point out that the previously recognized distinction between explicit comparison and implicit comparison (Kennedy 2009; Sawada 2009) does not fully exhaust the possible types of comparison constructions. The third type of comparison construction, which we call 'contrastive comparison', calls for a finer-grained classification that cross-classifies the three types of comparison constructions in terms of both semantic and pragmatic properties. We will then show that such a refined perspective straightforwardly becomes available once we extend the previous classification with a closer attention to discourse-structural properties of the relevant constructions in terms of the notion of Question under Discussion (Roberts 1996).
\end{abstract}

Keywords: comparative, implicit comparison, explicit comparison, contrastive comparison, comparison class, contrastiveness, Question under Discussion, Japanese

\section{Introduction}

The distinction between explicit comparison (EC) (1a) and implicit comparison (IC) (1b) has recently been recognized as an important cross-linguistic parameter of comparison constructions (cf, e.g., Kennedy 2009; Sawada 2009).

a. John is taller than Mary.

(explicit comparison)

b. $\{$ (As) compared to/with respect to $\}$ Mary, John is tall.

(implicit comparison)

Based on a range of distributional tests, Kennedy (2009) and Sawada (2009) argue for a typology of comparison constructions that distinguishes EC and IC, roughly along the following lines. EC establishes an ordering between two objects $x$ and $y$ with respect to gradable property $g$ using a morpho-syntactic form (i.e., degree

* We would like to thank Chris Kennedy, Marcin Morzycki, Osamu Sawada, Wataru Uegaki, and audiences at SALT 20 for valuable comments and suggestions. The first author is supported by the Research Fellowship of the Japan Society for the Promotion of Science under Grant No. 22-2912. 
morphology, -er in English) which semantically entails that $x$ is ordered above $y$ on the scale associated with $g$. IC, by contrast, establishes an ordering between two objects $x$ and $y$ only indirectly, by using a morpho-syntactic form (one without degree morphology, i.e., the positive form) which only makes a (non-gradable) judgment as to whether the object in question qualifies as having the (gradable) property $g$; the ordering between $x$ and $y$ is established pragmatically, by manipulating the context in such a way that the positive form is true of $x$ but false of $y$.

While the distinction between EC and IC has significantly clarified our understanding of the typology of comparison constructions, there are still some issues that have not yet been adequately addressed in the previous literature, among which are the following two important questions: what exactly are the semantic and pragmatic differences between EC and IC?; do EC and IC exhaust the possible types of comparison constructions? This paper aims to further our understanding of the typology of comparison constructions by first bringing to light a hitherto unnoticed third type of comparison construction in Japanese-dubbed here contrastive comparisonand then by providing a uniform analysis of the semantics and pragmatics of the three types of comparison constructions in terms of the notion of Question under Discussion (Roberts 1996). We will see that comparing the properties of the three constructions from this perspective contributes to the goal of answering the above two questions.

\section{Distributional properties of $\mathrm{EC}, \mathrm{IC}$ and $\mathrm{CC}$}

As noted by Kennedy (2009) and Sawada (2009), despite the fact that Japanese does not have overt comparative morphology, it clearly distinguishes between EC and IC. Specifically, the comparison construction with the yori phrase as in (2a) is a case of EC whereas the comparison construction with conditional clauses introduced by kurabe-\{tara/reba\} 'compared to' as in (2b) is a case of IC. We will review below a host of empirical facts that support this assumption. Japanese has yet another type of comparison construction, which is superficially similar to EC, exemplified by (2c).

a. John-wa Mary-yori se-ga takai.

John-TOP Mary-than height-NOM high

'John is taller than Mary.'

b. John-wa Mary-ni kurabe-\{tara/reba\} se-ga takai.

John-TOP Mary-DAT compare-if height-NOM high

'John is tall compared to Mary.'

(IC)

c. John-no-hoo-ga Mary-yori se-ga takai.

John-GEN-HOO-NOM Mary-than height-NOM high

'(Of the two,) John is taller than Mary.' (contrastive comparison; CC) 
Modes of comparison and Question under Discussion

We call this third type of comparison construction contrastive comparison (CC), for a reason that will become clear below. $\mathrm{CC}$ is similar to $\mathrm{EC}$ in that it involves an explicit yori phrase. As we will discuss in section 3, due to this morpho-syntactic property, the semantics of $\mathrm{CC}$ is essentially equivalent to that of EC. The two constructions differ in that CC marks the correlate of the yori phrase (by 'correlate' we mean the expression that denotes the object whose degree is compared with the degree introduced by the yori phrase) with the contrastive marker hoo, which has the function of contrasting two individuals salient in the discourse. Due to this contrastive marking, $\mathrm{CC}$ has a flavor that it shares with IC wherein the main point of assertion is to draw a distinction between the two objects being compared. CC apparently poses a challenge to the classification of comparison constructions into EC and IC since it exhibits a mixed property. Specifically, as we will see below, with respect to some tests, it behaves like EC, whereas with respect to some other tests, it behaves like IC. In the rest of this section, we review the distributional properties of the three types of comparison constructions. The relevant empirical generalizations are summarized in the following chart: ${ }^{1}$

\begin{tabular}{l|ccc} 
& EC & IC & CC \\
\hline crisp judgments & $\checkmark$ & $\#$ & $\checkmark$ \\
wazukani 'slightly' & $\checkmark$ & $\#$ & $\checkmark$ \\
minimum standard closed-scale predicates & $\checkmark$ & $\#$ & $\checkmark$ \\
negative implicature & $\#$ & $\checkmark$ & $\#$ \\
\hline measure phrases & $\checkmark$ & $\#$ & $\#$ \\
judgment enforcer & $\#$ & $\checkmark$ & $\checkmark$ \\
expectation reversal modifier yoppodo & $\#$ & $\checkmark$ & $\checkmark$
\end{tabular}

\subsection{Cases in which $\mathrm{CC}$ behaves like EC}

\subsubsection{Crisp judgments}

EC and IC contrast with one another in whether or not they allow for crisp judgments (Kennedy 2007), that is, whether they can be used for distinguishing between two objects when the degree difference between the two is very slight. CC behaves like EC in that it allows for crisp judgments. Thus, among the sentences in (2), (2a) and (2c) can be felicitously uttered in a context in which John is $180 \mathrm{~cm}$ tall and Mary is $179.5 \mathrm{~cm}$ tall, but (2b) cannot.

1 Crisp judgments, minimum standard closed-scale predicates, negative implicature and measure phrases are used by Kennedy (2009) for distinguishing between EC and IC. 


\subsubsection{Compatibility with wazukani 'slightly'}

Related to the above, EC allows for the degree modifier wazukani 'slightly' but IC doesn't. CC is compatible with wazukani, again, suggesting that, like EC, it is capable of distinguishing between two degrees that are very close to one another.

a. John-wa Mary-yori wazukani se-ga takai.

John-TOP Mary-than slightly height-NOM high

'John is slightly taller than Mary.'

b. ?? John-wa Mary-ni kurabe-\{tara/reba $\}$ wazukani se-ga takai. John-TOP Mary-DAT compare-if slightly height-NOM high

intended: 'John is slightly taller than Mary.'

c. John-no-hoo-ga Mary-yori wazukani se-ga takai.

John-GEN-HOO-NOM Mary-than slightly height-NOM high

'John is slightly taller of the two (= John and Mary).'

\subsubsection{Minimum standard closed-scale predicates}

Closed scale predicates with a minimum standard such as magat-te iru 'bent' are different from open scale predicates with a vague standard such as takai 'tall' in the way in which truth judgment is made. Judging the truth of 'A is bent' (the positive form) does not involve any vague standard, whereas judging the truth of 'A is tall' does involve a vague, contextually determined standard. As shown by the following data, EC and CC are compatible with minimum standard closed-scale predicates whereas IC is not. This again suggests that $\mathrm{EC}$ and $\mathrm{CC}$ contrast with IC in that the latter but not the former two makes reference to a contextually determined vague standard.

(5) a. Sao A-wa sao B-yori magat-te iru.

rod A-TOP rod B-than bent COP

'Rod A is more bent than rod B.'

b. ?? Sao A-wa sao B-ni kurabe-\{tara/reba\} magat-te iru.

rod A-TOP rod B-DAT compare-if bent COP

'??Rod A is bent compared to rod B.'

c. Sao A-no-hoo-ga sao B-yori magat-te iru.

rod A-GEN-HOO-NOM rod B-than bent COP

'Rod A is more bent of the two (= $\operatorname{rod} A$ and $\operatorname{rod} B)$.' 
Modes of comparison and Question under Discussion

\subsubsection{Negative implicature}

Finally, it is known that IC but not EC induces a certain implicature that the degree that the object in question possesses on the relevant scale is not very large (Kennedy 2009; Sawada 2009). For example, by saying Compared to Mary, John is tall (IC), there is a negative implicature 'John is not definitely tall (possibly borderline)'. As we will see below, this negative implicature for IC essentially arises from the fact that its semantics involves reference to a contextually determined, vague standard. CC behaves like EC in this respect as well in that it does not induce any such implicature, suggesting once again that its semantics is determined with respect to an explicit standard of comparison supplied by the yori phrase.

\subsection{Cases in which $\mathrm{CC}$ behaves like IC}

So far, we have seen that $\mathrm{CC}$ behaves like EC with respect to four phenomena, all uniformly suggesting that they contrast with IC in that their truth conditions are determined with respect to linguistically supplied, precise standards of comparison, rather than by making reference to contextually determined, vague standards. We will now review three phenomena in which the distribution of CC is the opposite, i.e., phenomena in which $\mathrm{CC}$ behaves like IC.

\subsubsection{Measure phrases}

As shown by the following examples, unlike EC but like IC, CC cannot occur with measure phrases.

$$
\begin{aligned}
& \text { a. John-wa Mary-yori 3-senti se-ga takai. } \\
& \text { John-TOP Mary-than 3cm height-NOM high } \\
& \text { 'John is 3cm taller than Mary.' } \\
& \text { b. ?? John-wa Mary-ni kurabe-\{tara/reba\} 3-senti se-ga takai. } \\
& \text { John-TOP Mary-DAT compare-if 3cm height-NOM high } \\
& \text { intended: 'John is } 3 \mathrm{~cm} \text { taller than Mary.' } \\
& \text { c. ?? John-no-hoo-ga Mary-yori 3-senti se-ga takai. } \\
& \text { John-GEN-HOO-NOM Mary-than 3cm height-NOM high } \\
& \text { intended: 'John is 3cm taller than Mary.' }
\end{aligned}
$$

This fact might seem somewhat puzzling given the observation in the previous section that the semantics of CC is essentially identical to that of EC. However, as we will argue in the next section, the incompatibility of measure phrases with CC can be explained from its pragmatic properties. 


\subsubsection{Judgment enforcer dotiraka-to ie-ba 'if anything'}

There are two other phenomena in which CC behaves like IC. First, Japanese has an expression dotiraka-to ie-ba, which literally translates as something like 'if one is (forced) to say which (of the two is the case)'. This expression is used for making a choice between two options under discussion and it indicates that the decision in question is hard to make. Given its pragmatic function, we call this expression 'judgment enforcer'. As shown in the following examples, the judgment enforcer dotiraka-to ie-ba is compatible with both IC and CC but not with EC.
a. ?? Dotiraka-to ie-ba John-wa Mary-yori se-ga takai. if anything John-TOP Mary-than height-NOM high 'If anything, John is taller than Mary.'
b. Dotiraka-to ie-ba John-wa Mary-ni kurabe-\{tara/reba\} if anything John-TOP Mary-DAT compare-if se-ga takai. height-NOM high 'If anything, John is tall as compared to Mary.'
c. Dotiraka-to ie-ba John-no-hoo-ga Mary-yori se-ga if anything John-GEN-HOO-NOM Mary-than height-NOM takai. high
'If anything, John is taller of the two (= John and Mary).'

\subsubsection{Expectation-reversal modifier yoppodo}

The adverb yoppodo presupposes that a certain negative expectation exists in the context which is countered by the assertion of the sentence. ${ }^{2}$ The contrast between the following examples illustrates the expectation-reversal property of yoppodo:

a. Mari/anata-wa Tanaka-san-ga yoppodo suki-nanda. Mari/you-TOP Tanaka-POL-NOM YOPPODO love-COP.EMPH

'Ah, (I didn't really realize it until now, but) Mari/you love(s) Mr. Tanaka so much.'

b. \# Watasi-wa Tanaka-san-ga yoppodo suki-nanda.

I-TOP Tanaka-POL-NOM YOPPODO love-COP.EMPH

intended: \# 'Ah, (I didn't really realize it until now, but) I love Mr. Tanaka so much.'

2 Cf. Sawada to appear for more details about the expectation-reversal property of yoppodo with scalar predicates. 
Modes of comparison and Question under Discussion

When the subject of the sentence is the second or third person, it is possible that the speaker has a certain, presupposed expectation about the mental state of that individual, which is then abandoned by the realization (expressed in the assertion of the sentence) that that expectation was incorrect. But if the subject is the first person, assuming, as would seem reasonable in most ordinary contexts, that people usually have accurate perceptions of their own mental states, such a correction of perceived mental state is impossible, making sentences like (8b) infelicitous. ${ }^{3}$

In comparison constructions, yoppodo is felicitous with IC and $\mathrm{CC}$ but not with $\mathrm{EC}$ :
a. ?? John-wa Mary-yori yoppodo se-ga takai.
John-TOP Mary-than YOPPODO height-NOM high
intended: '(Contrary to the expectation,) John is much taller than Mary.'
b. John-wa Mary-ni kurabe-\{tara/reba $\}$ yoppodo se-ga takai. John-TOP Mary-DAT compare-if YOPPODO height-NOM high
'(Contrary to the expectation,) John is rather tall as compared to Mary.'
c. John-no-hoo-ga Mary-yori yoppodo se-ga takai.
John-GEN-HOO-NOM Mary-than YOPPODO height-NOM high
'(Contrary to the expectation,) John is much taller than Mary.'

We will see below that the contrast among the three constructions here essentially arises from the ease with which an appropriate negative expectation to be presupposed by yoppodo can be invoked in the three construction given their respective pragmatic properties.

3 If this characterization of the meaning of yoppodo is correct, we expect that sentences like (8b) (with the first person subject) will improve in a context in which one realizes that one's own perception of one's mental state was inaccurate. This is indeed the case, as exemplified by the following example:

(i) [Context: The speaker suddenly realizes that she was in love with Mr. Tanaka by making a connection between her own (previously inexplicable) behavior and her (previously unrecognized) affection toward him.]

(Anna koto suru-nante) watasi-wa Tanaka-san-ga yoppodo suki-dat-ta-nda.

Such thing do-given I-TOP Tanaka-POL-NOM YOPPODO love-COP-PAST-EMPH

'Ah, (I didn't really realize it until now, but given that I did such a thing) I was in love with Mr. Tanaka so much.' 


\section{Analysis}

In the previous section, we have seen that $\mathrm{CC}$ exhibits a mixed distributional property wherein it behaves like EC in some respects but like IC in some other respects. The question that arises at the point is: why does $\mathrm{CC}$ behave the way it does? In particular, is it possible to analyze the semantics and pragmatics of the three types of comparison constructions in such a way that the distributional properties that we have reviewed above follow from some basic and independently motivated properties of the three constructions? We will argue below that such an analysis is in fact possible. The key to the solution for the problem, we will argue, comes from considering the discourse structural properties of these constructions in terms of the notion of Question under Discussion (Roberts 1996).

\subsection{Comparison constructions and Question under Discussion}

It might appear that the case of $\mathrm{CC}$ would pose a challenge for the previously recognized binary classification of comparison constructions into EC and IC. However, the challenge is only apparent, since the opposition between EC and IC is not really a simple binary distinction. Specifically, in order to understand the properties of of $\mathrm{CC}$, it becomes crucial to see that EC and IC contrast with one another along two dimensions, that is, in terms of both semantics and pragmatics, as has, at least implicitly, been recognized by Kennedy (2009) and Sawada (2009), and to investigate the pragmatic differences among the three constructions more systematically than has been done in previous work. The rest of this paper is devoted to precisely this investigation. To preview the conclusion, we will see that $\mathrm{CC}$ exhibits a mixed property since it shares the core semantic properties with EC and the core pragmatic properties with IC.

The pragmatic differences among the three constructions can most clearly be seen in question-answer pairs. As can be seen in (13), the three comparison constructions can serve as felicitous answers only to one of the three question types listed in (10)-(12) (specifically, degree question (10), polar question (11) and alternative question (12)). ${ }^{4}$

(10) John-wa dore-kurai se-ga takai-no?

John-TOP how much height-NOM tall-Q

'How tall is John?'

4 The contrast between IC and EC in terms of question-answer pairs like the ones given here is noted by Sawada (2009). 
Modes of comparison and Question under Discussion

(11) John-wa se-ga takai-no?

John-TOP height-NOM tall-Q

'Is John tall?'

John-to Mary-wa dotti-ga se-ga takai-no?

John-and Mary-TOP which-NOM height-NOM tall-Q

'Which is taller, John or Mary?'

\begin{tabular}{c|ccc} 
& $\mathrm{EC}(=(2 \mathrm{a}))$ & $\mathrm{IC}(=(2 \mathrm{~b}))$ & $\mathrm{CC}(=(2 \mathrm{c}))$ \\
\hline$(10)$ & $\checkmark$ & $\#$ & $\#$ \\
$(11)$ & $\#$ & $\checkmark$ & $\#$ \\
$(12)$ & $\#$ & $\#$ & $\checkmark$
\end{tabular}

The sharp contrast among the three constructions observed above suggests that these constructions are each associated with distinct discourse structural properties. Here, we will model these different discourse structural properties in terms of the notion of Question under Discussion (QUD; Roberts 1996), which most straightforwardly corresponds to the distributional properties of the three constructions in the question-answer pairs observed above, and, which, as we will see below, is a general enough notion for providing a principled explanation for the other relevant pragmatic properties of the three constructions that we will deal with below.

The idea behind the notion of QUD is that human communication involving language is a certain kind of game, where the goal of the game is to increase the amount of knowledge shared among interlocutors (see Roberts 1996 for a more complete discussion of the underlying ideas and a concrete proposal about how to formalize the relevant notions within a larger theory of discourse structure). Utterances are moves that players of the game (i.e., interlocutors) make, based on the strategies that they contrive for the purpose of achieving the global goal of increasing knowledge in the best possible way, given the current state of the game (i.e., what one believes to be true, what one believes one's interlocutor to believe to be true, what the current topic of the discourse is, etc.). QUDs can be thought of as the questions that guide the interlocutors in strategizing the moves that they make in this conversational game. Most typically, interlocutors make utterances in such a way that they provide (at least partial) answers to the current QUD, so that the common knowledge shared among the interlocutors (i.e., the set of questions whose answers have been resolved) increase as the discourse moves forward.

It is important to recognize at the outset that QUD is an inherently pragmatic notion and that the association between QUDs and sentence types is not one-to-one. What utterance responds to what QUD (and in what way it does so) is highly contextdependent. Also, in most typical discourse situations, there are multiple QUDs that are relevant, which, in Roberts's (1996) model, are hierarchically organized in a 
stack-like structure. It is nevertheless useful to talk about what one might call the 'default QUD' of some sentence type, to capture the tendency in which particular kinds of sentences are most typically used to respond to particular kinds of QUDs. Given the correspondences to the different question types observed above, this is clearly what is going on with the three types of comparison constructions under discussion, and we will therefore assume here that the default QUDs for EC, IC, and $\mathrm{CC}$ are the questions that they can felicitously respond to, as shown in chart (13).

\subsection{Semantics and pragmatics of EC, IC and CC}

Here, we will spell out our assumptions about the basic semantic and pragmatic properties of the three constructions. For the semantics of these constructions, we will adopt proposals in the previous literature (specifically, Kennedy 2007 and Sawada 2009 for EC and IC and Matsui \& Kubota 2010 for CC); for their pragmatics, we will pay special attention to how the more basic semantic and pragmatic properties of these constructions interact with one another to invoke the QUDs that they are each associated with.

For the semantics of gradable predicates, we assume a measure function-based approach (cf., e.g., Kennedy 1999, 2007), wherein gradable adjectives are taken to denote functions (of type $\langle e, d\rangle$ ) from individuals to degrees. ${ }^{5}$ In this approach, the denotation of the adjective takai can be given as in (14), which is a function that takes an individual as argument and returns a degree that this individual possesses on the scale that measures vertical length (i.e., $\operatorname{tall}(x)$ designates the degree to which $x$ is tall).

$$
[[\text { takai }]]=\lambda x \cdot \operatorname{tall}(x)
$$

The semantics of EC, i.e., the ordinary comparative, can then be formulated by giving the following denotation to the morpheme yori, which we take, following Sawada (2008) and Kennedy (2009), to have a function essentially equivalent to the comparative morpheme in English:

$$
[[\text { yori }]]=\lambda y \lambda g \lambda x \cdot g(x)>g(y)
$$

(15) says that yori takes two individuals $x$ and $y$ and a gradable property $g$ (of type $\langle e, d\rangle$ ) as arguments and asserts that the degree of $g$-ness of $x$ is larger than the degree of $g$-ness of $y$. Note that the height of the individual that yori takes as its first argument (i.e., $y$ ) fixes the standard of comparison explicitly in the semantics.

5 In the more standard approach, gradable adjectives are taken to denote relations between individuals and degrees. The choice of the measure function-based approach here is merely for expository ease; it enables a simpler formulation of the relevant aspects of compositional semantics for the kinds of phenomena that we deal with in this paper. 
Modes of comparison and Question under Discussion

With these assumptions, the denotation of (2a), a simple comparative sentence exemplifying EC, can be calculated as in (16), which asserts that the degree that John possesses on the scale of tallness is larger than the degree that Mary possesses, in other words, that John is taller than Mary.

$$
[[(2 \mathrm{a})]]=[[\text { yori }]]([[\text { John }]])([[\text { se-ga takai }])([[\operatorname{Mary}]])=\operatorname{tall}(\mathbf{j})>\operatorname{tall}(\mathbf{m})
$$

Crucially, this assertion of the sentence addresses the QUD associated with EC. The QUD 'How tall is John?' asks for the overall height of John. The assertion of (2a) provides a (partial) answer for it by specifying the lower threshold to John's height. ${ }^{6}$

We now turn to IC. IC is different from EC in that it does not involve overt comparative morphology (i.e., the morpheme - er in English and the yori phrase in Japanese). This suggests that IC is essentially a special case of positive assertion rather than a true comparative construction. More specifically, we assume, following Kennedy (2007) and Sawada (2009), that the semantics of IC is identical to that of the positive form of the adjective except that it restricts the domain of comparison down to the minimum size-containing only the two objects that are explicitly mentioned in the sentence.

To implement this idea, we first make the meaning of the positive form to be sensitive to the comparison class parameter $C$. This can be done by defining the pos operator, which is an empty operator that attaches to a gradable adjective of type $\langle e, d\rangle$ to produce an ordinary property of individuals (but one that is relativized to the comparison class parameter $C$ ) used in ordinary positive assertions with adjectives:

$$
[[\operatorname{pos}]]=\lambda g \lambda x \lambda C \cdot g(x) \geq \operatorname{stnd}(g)(C)
$$

With this definition of the pos operator, the meaning of the sentence John is tall can be calculated as follows:

6 Recall from above that, by 'the QUD' of EC, we do not necessarily mean the question that the sentence most directly addresses, but rather, the question that is typically the most central question in the context in which an EC sentence is uttered.

EC, of course, can respond to the more specific question 'Is John taller than Mary?' We nevertheless identify the more general degree question as the default QUD of EC, since the discourse structural properties of this construction can be better explained by making this assumption. Note that the two questions 'How tall is John?' and 'Is John tall?' stand in such a relation that the former is a super-question of the latter. The assertion of the EC sentence resolves the sub-question completely, thereby providing a partial answer to the more general QUD.

At least in Japanese, there is a clear contrast between EC and CC in the way in which they are associated with their respective QUDs. This is arguably related to the fact that, in Japanese, the subject of the EC sentence is marked by the topic marker -wa (and that that of the CC sentence is marked by the ordinary case marker $-g a$ ). Thus, the EC sentence ( $2 a$ ) is about (the height of) John, rather than about a comparison between John and Mary (in terms of height), which is what IC and CC are about. Given such an additional constraint on discourse structure, of the two questions that form the sub-super question pairs, only the more general question can be the real question under discussion that needs to be resolved in most typical situations in which an utterance of an EC sentence is made. 


$$
[[\text { John is tall }]]=[[\text { pos }]]([[\text { tall }]])([[\mathrm{John}]])=\lambda C \cdot \operatorname{tall}(\mathbf{j}) \geq \operatorname{stnd}(\operatorname{tall})(C)
$$

We assume that the value of the comparison class variable $C$ is determined contextually for simple assertions in the positive form.

As stated above, IC is different from ordinary positive assertion exactly in this respect. Specifically, we assume that the conditional clause introduced by kurabereba fixes the value of the comparison class parameter supplied for the stnd function as a two-membered set containing just the two individuals explicitly mentioned in the sentence. This can be done by specifying the meaning of kurabe-reba as in (19):

$$
[[\text { kurabe-reba }]=\lambda y \lambda g \lambda x \cdot g(x) \geq \operatorname{stnd}(g)(\{y, x\})
$$

With (19), the meaning of the IC sentence (2b) can be calculated as in (20):

$$
\begin{aligned}
{[[(2 \mathrm{~b})]] } & =[[\text { kurabe-reba }]]([[\text { Mary-ni }]])([[\text { se-ga takai }]])([[\text { John }]]) \\
& =\operatorname{tall}(\mathbf{j}) \geq \operatorname{stnd}(\operatorname{tall})(\{\mathbf{m}, \mathbf{j}\})
\end{aligned}
$$

The assertion of the IC sentence can be thought to provide a qualified answer to the QUD. The QUD for IC 'Is John tall?' asks whether John can be judged tall against a context-dependent, vague standard of tallness. (20) addresses this question by essentially saying that the QUD can be answered positively, on the condition that the domain of comparison is restricted to contain only John and Mary.

Finally, we analyze CC as a construction that has the same semantics as EC but has an additional pragmatic property that is similar to that of IC. Note first that CC differs from EC only in that the contrastive morpheme hoo is involved. As discussed by Matsui \& Kubota (2010), hoo is a marker that has the pragmatic function of invoking a contrast between two contextually salient objects. Following Matsui \& Kubota (2010), we assume the following denotation for $h o o:{ }^{7}$

7 Saying that the contextual variable $C$ designates the comparison class is actually a simplification, since hoo can appear in non-comparative sentences as well. The restriction that hoo introduces to the discourse should more appropriately be thought of as a kind of domain restriction, which happens to have the same effect as restricting the comparison class. See Matsui \& Kubota 2010: §5 for some discussion on this point. Note also that the notion of contrastiveness here is largely consistent with the notion of contrastive focus (CF) by Lee (2003). Thus, one might characterize hoo in Japanese as a morphological marker for CF. One possible difference, though, between the function of hoo and $\mathrm{CF}$ is that hoo does not by itself invoke the notion of exclusiveness (of alternative choices), which Lee (2003) takes to be a semantic characteristic of CF, as exemplified by the following sentence:

(i) [Choosing between two flavors of ice cream:]

banira-no-hoo-mo tyoko-no-hoo-mo oisisoo-na-node, mayot-te simau. vanilla-GEN-hoo-also chocolate-GEN-hoo-also tasty.looking-COP-because hesitate EMPH

'Since both the vanilla and chocolate flavors look tasty, I can't choose between the two.' 
Modes of comparison and Question under Discussion

$[$ hoo $]]=\lambda x: x \in C \wedge|C|=2 . x$

(where $C$ is the comparison class)

As can be seen in (21), hoo is truth conditionally vacuous (technically, it is an identity function $\lambda x . x$ ). From this, it straightforwardly follows that $\mathrm{CC}$ has the exact same truth conditions as EC. Since hoo is semantically vacuous, its effect on the meaning of the sentence is purely pragmatic. Specifically, it introduces the presupposition that the referent of the expression it attaches to is one of the elements in the comparison class and that the cardinality of the comparison class is two. This narrowing down of the comparison class is what makes $\mathrm{CC}$ have a similar pragmatic property as IC. With these assumptions, the CC example (2c) can be analyzed as follows:

a. Presupposition: $\mathbf{j} \in C \wedge|C|=2$

b. Assertion: tall $(\mathbf{j})>\operatorname{tall}(\mathbf{m})$

The presupposition of hoo (together with the assertion of the sentence which compares John and Mary on the same scale) is consistent with the QUD of CC, which is the alternative question 'Which is taller, John or Mary?' That is, the domain of comparison is restricted to the two individuals John and Mary and the question is which of the two stands out against the other on the relevant scale. Asserting that John is ordered above Mary on that scale against this background resolves the QUD since it then follows that John is the one who stands out against the other. This way, $\mathrm{CC}$ can be thought of as a construction that serves a pragmatic function similar to that of IC (distinguishing two individuals in terms of a scalar property) by means of a semantics that is identical to that of EC (explicit degree comparison).

\subsection{Accounting for the distributional properties}

Having clarified the basic semantic and pragmatic properties of the three kinds of comparison constructions, we are now ready to account for their distributional properties from section 2. For the contrast between EC and IC, we mostly build on the analyses proposed by Kennedy (2009) and Sawada (2009), with some extensions and reformulations where necessary so as to make them consistent with the QUDbased view of discourse structure that we adopt in this paper.

\subsubsection{Cases in which CC behaves like EC}

We start with cases in which $\mathrm{CC}$ behaves like EC. The fact that $\mathrm{CC}$, like EC but unlike IC, allows for crisp judgments and modification by wazukani 'slightly' straightforwardly follows from the fact that the semantics of CC is identical to that of EC: since the standard is fixed to the degree explicitly provided by the yori phrase, a slight 
difference in degree is enough for determining whether the object in question satisfies the standard. By contrast, such a situation is exactly what makes IC infelicitous. Since the predication in IC involves making reference to a vague standard, in order to differentiate the two objects being compared, there has to be a significantly larger degree difference between them.

The incompatibility of minimum standard predicates with IC essentially derives from the same reason. The function of IC is to manipulate the context in such a way that the vague standard for positive assertion is shifted, which affects the way in which truth conditional judgment is made with the gradable predicate in question. However, with minimum standard predicates like magat-te iru 'bent' in $(5 \mathrm{~b}, \mathrm{p} .4)$, narrowing down the comparison class to the minimum size does not have any semantic or pragmatic effect. This is so, because, with minimum standard predicates, the standard for truth conditional judgment targets the minimum endpoint of the scale regardless of the context. Thus, since $(5 \mathrm{~b})$ means exactly the same thing as the simpler positive assertion and the effect of the conditional clause introduced by kurabe-reba is totally vacuous, (5b) is pragmatically infelicitous. This kind of pragmatic infelicity does not arise with CC. Unlike IC (and like EC), in the semantics, CC purely compares the degrees that the two objects possess on the relevant scale. Thus, regardless of whether the scale is open-ended or closed, the sentence is equally informative.

Finally, the presence vs. absence of the negative implicatures in IC on the one hand and EC and CC on the other is due to the different pragmatic properties of the three constructions, which in turn derive from their fundamental semantic difference as to whether predication in terms of a contextually determined vague standard is involved. Sawada (2009) investigates in detail the way in which the negative implicature arises in IC given its semantic and pragmatic properties. We here recast the core aspects of his proposal within the present, QUD-based setup. Recall from above that the QUD of IC is the polar question 'Is John tall?' The assertion of the IC sentence responds to this QUD by providing a qualified positive answer to it, specifically, by saying that John can be regarded tall on the condition that the domain is restricted to contain only John and Mary. Crucially, given this semantic and pragmatic property of the construction, it follows that an assertion with IC is felicitous only if both of the following conditions are met:

(i) restricting the domain this way has the effect of lowering the standard

(ii) lowering the standard has the effect of making the (otherwise false) positive answer to the QUD true

(i) has to be satisfied since, if (i) weren't the case, restricting the domain with the use of IC would not have any useful effect for the purpose of answering the QUD 
positively. And given that (i) obtains, it further follows that (ii) is also the case, since, again, if it weren't, a simple positive assertion would suffice (and would be more informative and appropriate) as an answer to the QUD. But saying that condition (ii) holds is the same as saying that John cannot be regarded tall by using the ordinary standard, which is nothing other than the negative implicature that is felt to be present in the assertion of IC.

With EC and CC, this kind of negative implicature does not arise simply because the standard of comparison is explicit for these constructions. Since no reference is made to the contextually determined standard to begin with, there could possibly be no way in which any implicature associated with that vague standard would arise.

\subsubsection{Cases in which $\mathrm{CC}$ behaves like IC}

The cases in which $\mathrm{CC}$ behaves like IC all point to a pragmatic difference between EC on the one hand and IC and CC on the other such that the essential property of $\mathrm{EC}$ is degree measurement whereas IC and CC are constructions for making some kind of binary judgments for the purpose of resolving the QUD.

First, the distribution of measure phrases with these constructions can be accounted for as follows. The function of measure phrases is to specify the amount by which the object in question is located above the standard on the relevant scale. This perfectly matches the pragmatic function of EC whereby it responds to its QUD, which asks for the degree that the object in question possesses on the scale.

By contrast, the meanings of measure phrases are not compatible with the pragmatic functions of IC and CC. The QUDs of IC and CC are a polar question and an alternative question, respectively. Both of these questions demand making some kind of binary judgments: in the case of a polar question, whether or not the proposition posed as the question is true, and in the case of an alternative question, which of the two individuals salient in the discourse satisfies the property in question. In responding to such questions by making predications with gradable predicates, what is crucial is whether the object in question does or does not satisfy the standard, irrespective of whether the standard is vague or precise. But then, specifying the exact amount by which the object in question exceeds the standard is irrelevant for the purpose of answering the QUD and this is why measure phrases are incompatible with IC and CC. ${ }^{8}$

Second, the judgment enforcer dotiraka-to ie-ba is an expression that indicates

8 This leads one to expect that when such an amount is relevant in the context, the acceptability of the sentence is improved. The following example corroborates this prediction:

(i) Q: John-to Mary, dotti-no-hoo-ga doredake se-ga takai-no? John-and Mary which-GEN-HOO-NOM how much height-NOM high-Q 'Which is taller, John or Mary, and how much?' 
that the assertion of the sentence addresses a binary judgment that is subtle and difficult to make. This fits with the pragmatic properties of IC and CC, given that both address QUDs that ask for making some kind of binary judgment. For these constructions, the addition of dotiraka-to ie-ba to the sentence has the effect of signaling that the particular QUD that the sentence addresses is difficult to resolve. By contrast, the QUD of EC, being a degree question, is not associated with any particular binary judgment. Thus, the pragmatic function of the construction is not consistent with that of the judgment enforcer, and, for this reason, the use of dotiraka-to ie-ba with EC is infelicitous.

Finally, the distribution of the expectation reversal modifier yoppodo can be accounted for along lines similar to the case of the judgment enforcer dotiraka-to $i e-b a$. The expectation reversal function of yoppodo arises from an interaction of its presupposition and assertion. Specifically, we assume that yoppodo presupposes that the answer to the QUD is expected to be negative and that sentences of the form yoppodo $\phi$ asserts $\phi$ and is felicitous if and only if $\phi$ counters that expectation.

This analysis of yoppodo predicts its distributions in the three types of comparison constructions appropriately. Again, the key property that distinguishes EC and the other two constructions is their difference in discourse structural properties. In the case of IC and CC, for which the QUD is a question that demands a binary judgment, the presupposition of yoppodo can be satisfied naturally: the negative expectation invoked by yoppodo can simply be identified with the opposite judgment countered by the assertion of the sentence. More specifically, with IC, the use of yoppodo indicates that the answer to the QUD is expected to be negative and the assertion of the sentence counters that expectation by providing a positive (albeit qualified) answer to it. Similarly, with CC, the use of yoppodo suggests the existence of a prevailing expectation (for (9c), this is the expectation that Mary, and not John, is the taller of the two), which again, is countered by the assertion of the sentence. $\mathrm{EC}$, by contrast, is crucially different from IC and CC in that its QUD is a degree question, which is not associated with any particular binary judgment. Given this, there is no plausible candidate for the negative expectation to be presupposed by yoppodo and the sentence becomes infelicitous due to presupposition failure. ${ }^{9}$

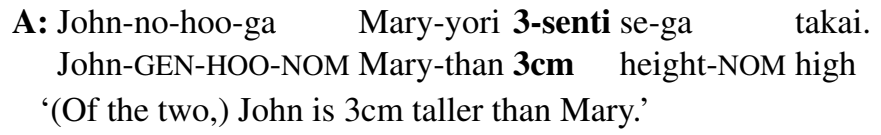

9 Just like the other cases considered in this section, the incompatibility of EC and yoppodo is due to the general pragmatic function of the former and the specific condition that needs to be satisfied with the use of the latter, namely, its negative expectation presupposition. Thus, if the presupposition of yoppodo is satisfied in some way or other, the incompatibility of EC and yoppodo goes away, as exemplified by the following sentence (thanks to Wataru Uegaki (p.c.) for this example): 
Modes of comparison and Question under Discussion

\section{Conclusion}

This paper started with the observation that comparative sentences with the contrastive marker hoo in Japanese (contrastive comparison; CC) poses an apparent challenge to the previously recognized typology of comparison constructions in terms of explicit comparison (EC) and implicit comparison (IC). Specifically, we have presented a set of data that show that $\mathrm{CC}$ exhibits mixed distributional properties wherein it sometimes behaves like EC and sometimes like IC. We then proposed an analysis of these constructions by extending the previous classification of EC and IC with a closer examination of the discourse structural properties of the three constructions in terms of the notion of Question under Discussion (Roberts 1996).

The picture that has emerged from this reconsideration of the typology of comparison constructions cross-classifies the three types of comparison constructions in terms of semantic and pragmatic properties along the following lines:

\begin{tabular}{|c|c|c|}
\hline pragmatics semantics & explicit standard & implicit standard \\
\hline $\begin{array}{l}\text { measuring degrees } \\
\text { making a binary judgment }\end{array}$ & $\begin{array}{l}\mathrm{EC} \\
\mathrm{CC}\end{array}$ & $\begin{array}{l}\text { (not attested) } \\
\text { IC }\end{array}$ \\
\hline
\end{tabular}

Looking at this chart, one might wonder why the top right corner is unattested. That this slot is empty is most likely not just an accident: it simply does not make sense to measure degrees with implicit, contextually determined vague standard. So, we now have an answer to the question that we posed above, namely, the question of why such a thing as $\mathrm{CC}$ exists in addition to EC and IC.

We would like to conclude the discussion with a brief remark on the wider implications of the results obtained in this paper. As discussed by Kennedy (2007), using an implicit standard for making a comparison of two objects necessitates restricting the domain of comparison down to the size of two so that the vague predicate can be used to distinguish between the two relevancy objects. The existence of CC in Japanese suggests that such pragmatic domain restriction can have an effect in cases in which an explicit standard is involved as well. The three-way

(i) John-wa Mary-nanka-yori yoppodo se-ga takai.

John-TOP Mary-EMPH-than YOPPODO height-NOM high

'(Contrary to the expectation, ) John is much taller than Mary.'

Here, the pejorative emphatic particle nanka, attached to Mary, introduces the implicature that Mary is not very tall. This further invokes the implicature that, given that Mary is chosen as a contextually meaningful standard of comparison, John isn't particularly tall either. This latter implicature can be taken as a prevailing negative expectation which satisfies the presupposition of yoppodo. In a sense, the pejorative emphatic particle nanka invokes an implicature similar to that in IC and the contrast between EC and IC is neutralized for that reason. 
classification of comparison constructions in Japanese that we have proposed in this paper recognizes the parameter of contrastiveness as a separate pragmatic factor that is in principle independent of the previously recognized semantic parameter of standard of comparison. To what extent this refined classification is plausible of course depends on its wider cross-linguistic applicability; the question to be investigated is whether similar patterns as is observed in Japanese can be found in different types of languages - those in which contrastive marking is done in overt morpho-syntax like Japanese and those in which it is done otherwise (for example, prosodically, as in English).

\section{References}

Kennedy, Christopher. 1999. Projecting the adjective: The syntax and semantics of gradability and comparison. New York: Garland Press.

Kennedy, Christopher. 2007. Vagueness and grammar: The semantics of relative and absolute gradable adjectives. Linguistics and Philosophy 30(1). 1-45. doi:10.1007/s10988-006-9008-0.

Kennedy, Christopher. 2009. Modes of comparison. In Proceedings from the Annual Meeting of the Chicago Linguistic Society, vol. 43, 141-165.

Lee, Chungmin. 2003. Contrastive topic and/or contrastive focus. In William McClure (ed.), Japanese/Korean Linguistics, vol. 12, Stanford: CSLI Publications.

Matsui, Ai \& Yusuke Kubota. 2010. Comparatives and contrastiveness: Semantics and pragmatics of Japanese hoo comparatives. In Proceedings of Formal Approaches to Japanese Linguistics 5, Cambridge, MA: MITWPL.

Roberts, Craige. 1996. Information structure in discourse: Towards an integrated formal theory of pragmatics. In Jae-Hak Yoon \& Andreas Kathol (eds.), Papers in semantics, vol. 49 Ohio State University Working Papers in Linguistics, Department of Linguistics, The Ohio State University.

Sawada, Osamu. 2008. The comparative morpheme in Modern Japanese. In Proceedings of the 34th Annual Meeting of the Berkeley Linguistics Society, Berkeley, CA: Berkeley Linguistics Society.

Sawada, Osamu. 2009. Pragmatic aspects of implicit comparison: An economy-based approach. Journal of Pragmatics 41. 1079-1103. doi:10.1016/j.pragma.2008.12.004.

Sawada, Osamu. to appear. Modes of scalar reversal in Japanese. In Japanese/Korean Linguistics, vol. 17, Stanford: CSLI Publications. 
Modes of comparison and Question under Discussion

Yusuke Kubota

University of Tokyo, JSPS

yk@phiz.c.u-tokyo.ac.jp
Ai Matsui

Michigan State University

matsuiai@msu.edu 